\title{
Palisading cells of rheumatoid nodules: comparison with synovial intimal cells
}

\author{
J C W Edwards, L S Wilkinson, A A Pitsillides
}

\begin{abstract}
Objectives-The palisading cells of rheumatoid nodules share certain features with synovial intimal cells. The similarities between the two cell populations have been reassessed using new cytochemical markers.

Methods-Cell populations in cryostat sections of non-inflamed, rheumatoid and osteoarthritic synovial tissues, and rheumatoid nodules were assessed for the presence of CD68, prolyl hydroxylase, vascular cell adhesion molecule 1 (VCAM1), and the $\alpha 4$ and $\beta 1$ integrin chains, and the activity of uridine diphosphoglucose dehydrogenase (UDPGD) and nonspecific esterase.
\end{abstract}

Results-Synovial intimal cells formed a dual population of macrophages (nonspecific esterase positive, strongly positive for CD68) and fibroblastic cells (prolyl hydroxylase positive). The latter showed prominent VCAM-1 expression and high UDPGD activity as previously reported and also prominent $\beta 1$ integrin chain expression. Palisading cells similarly proved to be a dual population of macrophages and fibroblastic cells. In contrast with synovial intima, however, the fibroblastic cells lacked UDPGD activity and expression of VCAM-1 and showed no preferential expression of the $\beta 1$ integrin chain. The exception to this rule was where nodules contained central clefts, which were lined with cells showing all the features associated with synovial intimal cells.

Conclusion-Palisading cells are a mixture of macrophages and fibroblasts, but the latter show no evidence of synoviocyte differentiation. Cells with features of synoviocytes may occur lining clefts within areas of necrobiosis.

(Ann Rheum Dis 1993; 52: 801-805)

Group,

Division of

Rheumatology,

University College

London Medical

School, London,

United Kingdom

J C W Edwards

LS Wilkinson,

L A Pitsillides

Correspondence to:

DrJ C W Edwards,

Arthur Stanley House,

Tottenham Street,

London W1P 9PG

United Kingdom.

Accepted for publication 21 July 1993 flattened in the plane of the surface, ${ }^{8}$ whereas nodule palisading cells form an interface between living and necrotic tissue and are elongated at right angles to the plane of the interface. ${ }^{7}$ The two populations, however, contain macrophages and are arranged in a closely packed layer. Fassbender has pointed out that synovial intimal cells in rheumatoid arthritis can blend continuously with typical palisading cells. ${ }^{1}$ This is commonly seen when they are overlain by necrotic debris containing fibrin. Conversely, nodules are commonly associated with synovial bursae and, in addition, may contain central clefts, which may be partially lined by cells with the flattened appearance of synovial intimal cells.

Cytochemical and immunochemical analysis of the two populations of cells has tended to focus on macrophages. It has become increasingly clear, however, that not all the cells, either in synovial intima or in the palisading layer of nodules, carry leucocyte related markers. In synovium, studies suggest a clear cytochemical distinction between macrophages and unrelated fibroblast-like cells, corresponding to the two populations defined by ultrastructure as type A and type B. ${ }^{9}$ Moreover, the fibroblast-like cells, perhaps best referred to as true synoviocytes, show cytochemical features which distinguish them from other fibroblasts, including the prominent expression of vascular cell adhesion molecule 1 (VCAM-1) $)^{1011}$ and high activity of the enzyme uridine diphosphoglucose dehydrogenase (UDPGD). ${ }^{9}$ Despite the fact that nodule palisading cells were at one time referred to as fibroblasts (on traditional morphological grounds) the presence of cells with cytochemical characteristics of fibroblasts in the palisading layer is not well documented.

The aim of this study was to make a detailed comparison between synovial intimal cells and the palisading cells of rheumatoid nodules to establish whether these two populations can be considered equivalent in terms of cytochemistry, and, by inference, their possible role in the genesis of inflammation in rheumatoid disease. Particular emphasis was given to fibroblast related markers, including the content of prolyl hydroxylase, an enzyme with a role in collagen synthesis, the activity of UDPGD, with a role in hyaluronan synthesis, and the content of VCAM-1. A number of macrophage and leucocyte related markers were also used. No ideal macrophage marker exists, but prominent non-specific esterase activity, in the context of connective tissue, has high specificity. It does not mark immature macrophages well, but it is sensitive in the context of synovial intimal macrophages, which appear to be of mature phenotype. ${ }^{12} \mathrm{~A}$ high content of CD68 is a sensitive marker of tissue macrophages. ${ }^{13}$ It may be less specific 
than non-specific esterase activity. CD68 is associated with lysosomes and is also prominent in other cell types which contain numerous lysosomes, such as osteoclasts. Low levels of CD68 may be present on fibroblastlike synoviocytes, ${ }^{9}$ but not levels comparable with those seen on macrophages. Used in parallel non-specific esterase activity and CD68 content provide the best evidence of macrophage identity in the context of mononuclear cells in inflamed connective tissue. The presence of the VCAM-1 ligand VLA-4 was also assessed using antibodies to the $\alpha 4$ and $\beta 1$ chains of this integrin heterodimer.

\section{Materials and methods \\ TISSUES \\ Synovial tissues}

Non-inflamed synovium was obtained from the limbs of five patients undergoing amputation for sarcomata. Tissues were taken from clinically normal areas at least $20 \mathrm{~cm}$ distant from the tumour, and showed no histological evidence of disease. Inflamed synovium was obtained from patients with osteoarthritis or rheumatoid arthritis (definite or classical) undergoing arthroplasty and was obtained within 20 minutes of removal.

\section{Rheumatoid nodules}

Rheumatoid nodules were obtained from the subcutaneous tissues of nine patients with rheumatoid arthritis after excision for symptomatic reasons.

\section{TISSUE PROCESSING}

Tissues were trimmed of fat, dissected into 3-5 $\mathrm{mm}$ pieces, snap frozen in $\mathrm{n}$-hexane at $-70^{\circ} \mathrm{C}$ and stored at $-70^{\circ} \mathrm{C}$ until required for sectioning. Sections were cut at $-35^{\circ} \mathrm{C}$ on a Bright's cryostat and air dried for 30 minutes before cytochemical/immunochemical analysis.

\section{UDPGD ACTIVITY}

Uridine diphosphoglucose dehydrogenase activity was assessed using the method of Mehdizadeh et al. ${ }^{14}$ Freshly cut $7 \mu \mathrm{m}$ sections were incubated for 20 minutes at $37^{\circ} \mathrm{C}$ with a reaction medium consisting of uridine diphosphoglucose and nicotinamide adenine dinucleotide in glycyl glycine buffer at $\mathrm{pH} 7.8$ containing $30 \%$ polyvinyl alcohol and saturated with nitrogen to which nitro blue tetrazolium was added. Control preparations were incubated with substrate free medium. Sections were washed in water, and mounted in Aquamount (BDH), or, for subsequent immunostaining, washed in phosphate buffered saline (PBS). Preparations for double labelling were fixed in acetone for five minutes before reacting for enzyme activity. Unfixed controls ensured activity was not lost during fixation.

NON-SPECIFIC ESTERASE ACTIVITY

Non-specific esterase activity was assessed using $\alpha$-naphthyl acetate as described previously. ${ }^{12}$ When assessment of non-specific esterase activity was combined with assessment of UDPGD activity or immunochemistry, assessment of non-specific esterase activity was performed last.

\section{IMMUNOCHEMISTRY}

The following monoclonal antibodies were used for the characterisation of synovial intimal and nodule palisading cells: EBM11 (antiCD68) for macrophages ${ }^{13}$ at $1 / 200$ (DAKO); 5B5 (anti-prolyl hydroxylase) for fibroblasts ${ }^{15}$ at $1 / 50$ (DAKO), $1.4 \mathrm{C} 3$ for VCAM-1, ${ }^{16} \mathrm{HP} 2 /$ 1 for the $\alpha 4$ integrin chain ${ }^{17}$ at $1 / 200$ (Serotec), and B-D15 for the $\beta 1$ integrin chain ${ }^{18}$ at $1 / 50$ (Serotec). In addition, the following antibodies were used to identify other leucocyte populations: UCHT1 (anti-CD3) for $\mathrm{T}$ lymphocytes (DAKO), 4KB128 (anti-CD22) for B lymphocytes, and NP57 (antineutrophil) elastase for neutrophils. Antibodies were used in either native form or biotinylated for double labelling. Binding of monoclonal antibodies was visualised using either goat antimouse IgG/fluorescein isothiocyanate (FITC) (Sera Lab) at $1 / 20$ in PBS plus $20 \%$ normal human serum (NHS) or, for biotinylated monoclonal antibodies, streptavidin/tetramethyl rhodamine isothiocyanate (TRITC) (Sera Lab) at $1 / 20$ in PBS plus $20 \%$ NHS, or, for permanent enzymatic preparations, rabbit antimouse immunoglobulins (DAKO) $1 / 20$ in TRIS buffered saline (TBS) plus $20 \%$ NHS and alkaline phosphatase antialkaline phosphatase (DAKO) at $1 / 50$ in TBS plus $20 \%$ NHS.

Sections were incubated with primary reagents for one hour at room temperature, and all subsequent incubations were for 30 minutes. Incubations for immunofluorescence were in PBS at $\mathrm{pH} 7 \cdot 6$, and for the alkaline phosphatase-antialkaline phosphatase (APAAP) technique in TBS at $\mathrm{pH} 7 \cdot 8$ Specimens for immunofluorescence were mounted in glycerol containing 1,4-diazabicyclo[2.2.2] octane (Sigma), and specimens stained using APAAP in Aquamount (BDH).

\section{DOUBLE LABELLING}

Initially, serial sections were assessed for UDPGD and non-specific esterase activity and the presence of CD68, VCAM-1, $\alpha 4$ and $\beta 1$ integrin chains, and prolyl hydroxylase using alkaline phosphatase-antialkaline phosphatase conjugated second antisera and fast red, with haematoxylin as counterstain. For those markers giving positive staining, double labelling was performed to establish the cell populations involved. All double labelling procedures were controlled by comparison with single labelled serial sections. Assessment of UDPGD activity was combined with immunochemical staining for CD68, prolyl hydroxylase, VCAM-1, and $\beta 1$ integrin. A fluoresceinated second antibody was used. Assessment of non-specific esterase activity was combined with immunochemical staining for prolyl hydroxylase, VCAM-1, and $\beta 1$ 

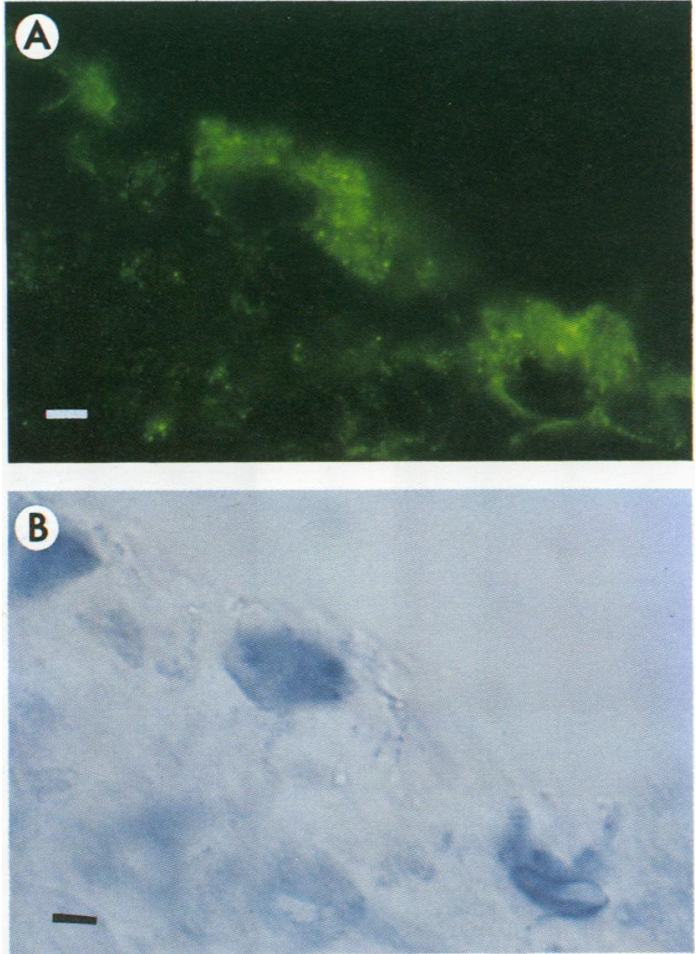

Figure 1 Rheumatoid synovium double labelled for $(A)$ $\beta 1$ integrin (green) and (B) uridine diphosphoglucose dehydrogenase (UDPGD) activity (blue). A mesh of staining for $\beta 1$ integrin is present with the brightest staining on the luminal aspect of cells of high UDPGD activity. Bar $=10 \mu \mathrm{m}$

integrin using fast blue as substrate. Double immunofluorescent staining for CD68/ VCAM- 1 and CD68/ $\beta 1$ integrin was achieved by successive incubation with one primary antibody, antimouse IgG FITC, $20 \%$ normal mouse serum, the second (biotinylated) primary antibody, and streptavidin TRITC.

\section{Results}

SYNOVIAL TISSUES

Findings on normal and diseased synovial tissues were in keeping with those previously published. ${ }^{9}{ }^{1019}$ By a series of double labelling

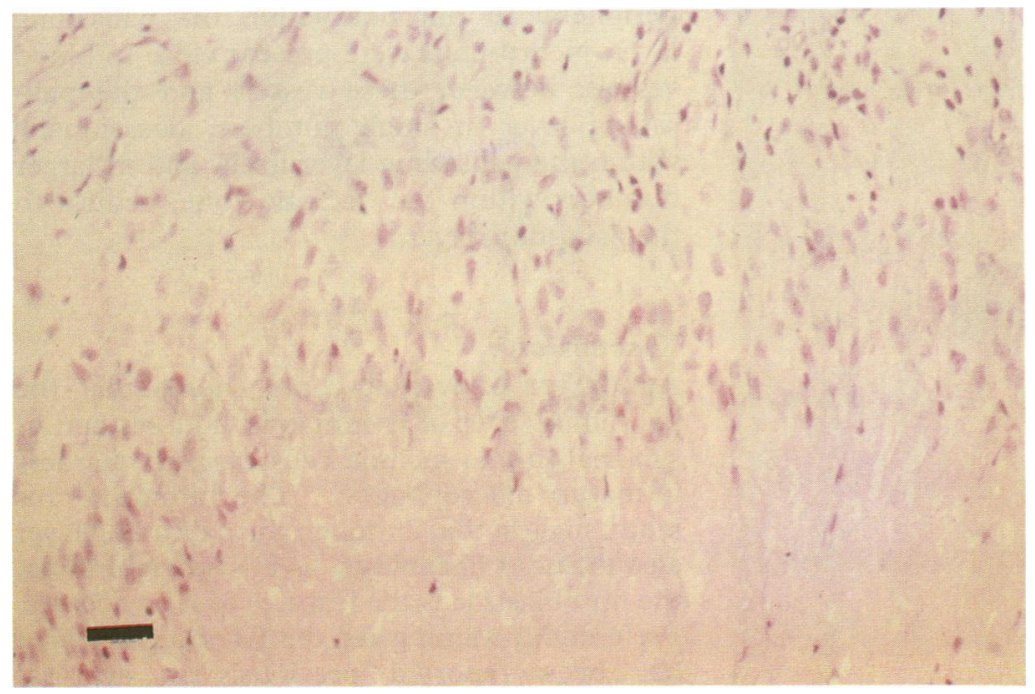

Figure 2 Rheumatoid nodule stained with haematoxylin and eosin, showing amorphous pink necrobiotic material (lower edge of micrograph) surrounded by a layer of palisading cells. Bar $=40 \mu \mathrm{m}$. experiments it was shown that normal synovial intimal cells could be divided into $(a)$ macrophages positive for CD68 or nonspecific esterase and $(b)$ synoviocytes of high UDPGD activity, VCAM-1 expression, and prolyl hydroxylase content. The proportions of the two cell types varied from place to place within samples and between samples. In normal and osteoarthritic tissues the ratio of mean UDPGD activity in synoviocytes to that in subintimal cells, assessed by microdensitometry, was consistently greater than $4: 1$ (highest value 9:1). In rheumatoid synovial tissues levels of UDPGD activity in intimal cells tended to be lower with activity ratios (synoviocyte: subintimal cell) of $2 \cdot 5: 1$ or less.

The $\alpha 4$ integrin chain was found on cells distributed widely in normal and diseased synovium, as expected from the presence of macrophages or T lymphocytes, or both. There was little or no staining of intimal cells, however. In contrast, $\beta 1$ integrin was preferentially expressed on cells in the intimal layer. Staining for $\beta 1$ integrin formed a mesh throughout the intimal layer, but the brightest staining was seen on the luminal surface of cells of high UDPGD activity (fig 1).

Lymphocytes and neutrophils were not present in the intimal layer. $T$ and $B$ lymphocytes were present in the deeper layers of diseased tissues. Neutrophils were present in small numbers in rheumatoid tissues between the most superficial venules and the tissue surface and in larger numbers within deposits of fibrin on the tissue surface.

RHEUMATOID NODULES

As with synovial intimal cells, the palisading cells of rheumatoid nodule (fig 2) could be divided into (a) macrophages positive for CD68 or non-specific esterase or (b) prolyl hydroxylase positive fibroblasts (fig 3). The macrophages were in the greater number and like synovial intimal macrophages showed the strongest non-specific esterase activity of any cells in the tissue. A certain amount of overlap of low level staining was seen with CD68 and prolyl hydroxylase. This has been seen previously with intimal cells and probably represents (a) the presence of small amounts of the lysosome related protein CD68 in fibroblasts and $(b)$ phagocytosis of fibroblast cytoplasmic debris by macrophages. Nevertheless, double labelling indicated that all cells could be clearly assigned to one or the other type.

In contrast with synovial intimal cells UDPGD activity and VCAM-1 expression in palisading cells were minimal. The UDPGD activity in palisading cells was no higher than that in surrounding fibroblasts and, indeed, substrate specific activity in the presence of substrate was not measurably greater than the activity shown with substrate free incubation medium (i.e. measurable substrate specific activity $=0$; no activity ratio obtainable). The few weakly VCAM-1 positive cells in the palisade showed levels of staining no higher than that seen on cells scattered in other areas of the tissue. 


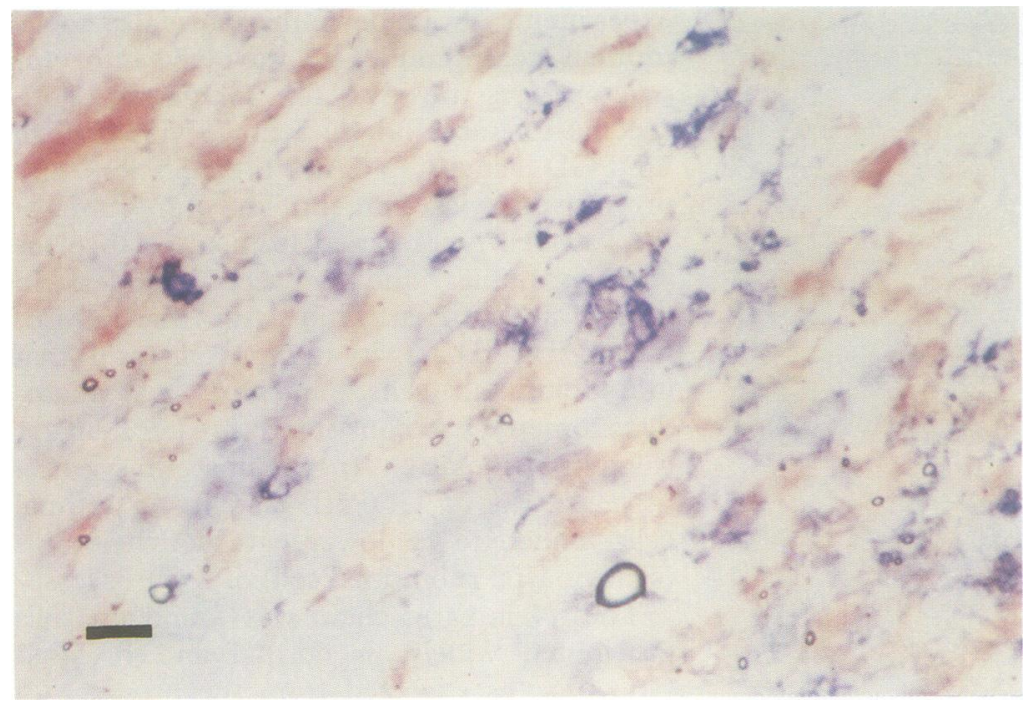

Figure 3 Palisading cells in a rheumatoid nodule double labelled for non-specific esterase activity (red/brown) and prolyl hydroxylase content (blue) showing a mixed population. Bar $=20 \mu \mathrm{m}$.
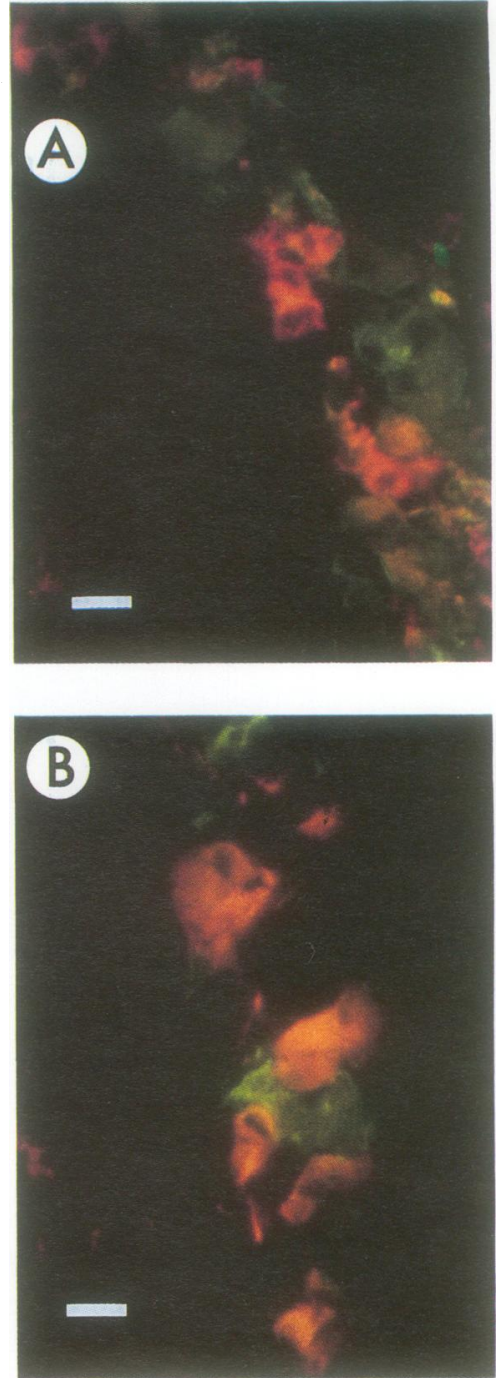

Figure 5 (A) Rheumatoid synovium and (B) cells lining a cleft within the necrotic centre of a rheumatoid nodule, double labelled for CD68 (red) and vascular cell adhesion molecule 1, (green) showing a similar mixture of cell types. $B a r=20 \mu \mathrm{m}$.

generally, with no preferential staining of the palisading layer. In contrast with synovium, staining for $\beta 1$ integrin was generally weak, with no preferential staining of the palisading layer.

Lymphocytes were present in small clusters peripheral to but not within the palisading layer. Neutrophils were present close to blood vessels, between these vessels and the palisading layer in small numbers, occasionally within the palisading layer itself, and in larger numbers within the necrotic zone, central to the palisading layer.

\section{Discussion}

A number of workers have shown that the palisading layer of rheumatoid nodules and of synovial intima include cells carrying markers of mature macrophages. ${ }^{2-6}$ The findings of this study suggest that although the palisading layer also includes fibroblasts, as in synovial intima, the fibroblasts lack the features of synoviocytes. Preferential staining for the $\beta 1$ integrin chain has been reported previously in synovial intima. ${ }^{20}$ The present double labelling studies suggest that the preferential staining in the intima is due to the presence of the $\beta 1$ chain
Figure 4 Cells lining a cleft within the necrotic centre of a rheumatoid nodule assessed for uridine diphosphoglucose dehydrogenase activity. Cells of high activity are seen confined to the surface. Bar $=20 \mu \mathrm{m}$. 
on synoviocyte membranes, chiefly on processes on the luminal side of the cells. In the absence of staining for the $\alpha 4$ chain it is likely that the $\beta 1$ chain on synoviocytes is present in the form of VLA-5. The $\beta 1$ integrin chain does not appear to be present on either type of cell in the palisading layer of nodules. We have confirmed that macrophages of the palisading layer share the prominent non-specific esterase activity of synovial intimal macrophages.

The appearance of VCAM-1 and high UDPGD activity on cells lining a cleft within an area of necrobiosis would seem to be a late secondary event, probably of no significance to the genesis of the original lesion. VCAM-1 expression and UDPGD activity have also been found in cells on the surface of regenerated synovial tissue taken from around an artificial joint prosthesis. ${ }^{21}$ Evidence is accumulating that fibroblastic cells will only take on these properties when present on connective tissue surfaces exposed to shearing stresses. ${ }^{22}$ This may be relevant to the absence of synoviocytes from the palisading layer itself. Nevertheless, as nodules characteristically form at sites of mechanical stress, shearing forces may well be experienced by cells on the surface of clefts at the lesion centre.

It has been suggested that the cellular events in synovitis and the rheumatoid nodule are essentially the same. Infiltrating lymphocytes appear to remain largely on the vascular side of the macrophage/fibroblast layer where they may form clusters. Neutrophils appear to migrate rapidly through this layer so that they are found at greatest density either in synovial fluid or in the necrotic centre of the nodule. The synovial intima and the palisading layer could be seen as having a similar role in perpetuating the local inflammatory process via the generation of chemotactic gradients, angiogenic factors, and other mediators. The findings of this study do not alter this possibility, but it appears that the specialised nature of synoviocytes is not critical to any cooperative role they might have with the associated macrophages in disease. The expression of VCAM-1 in particular does not appear to be essential to such a role in the nodule, though it remains possible that VCAM-1 expression in synovium facilitates interactions, thereby making the joint more susceptible to inflammation in the presence of a systemic immunological disturbance, with almost $100 \%$ of rheumatoid patients having joint disease and only $20 \%$ having subcutaneous nodules.
This study was made possible by the financial support of the Arthritis and Rheumatism Council, which is gratefully acknowledged. Antibody 1.4C3 for VCAM-1 was kindly supplied by Dr D O Haskard.

1 Fassbender H G. Rheumatoid arthritis. In: Pathology of rheumatic diseases. Berlin: Springer, 1975: 118-9.

2 Edwards J C W. Structural and microscopic changes. In: Henderson B, Edwards J C W, eds. The synovial lining in health and disease. London: Chapman and Hall, 1987: 266-70.

3 Athanasou A, Quinn J, Woods C G, McGee J O'D. Immunohistology of rheumatoid nodules and rheumatoid synovium. Ann Rheum Dis 1988; 47: 398-403.

4 Duke O, Hobbs S, Panayi G S, Poulter L W, Rasker J J, Janossy G. A combined immunohistological and histochemical analysis of lymphocyte and macrophage subpopulations in the rheumatoid nodule. Clin Exp Immunol 1984; 56: 239-46.

5 Hedfors E, Klareskog L, Lindblad S, Forsum U, Lindahl G. Phenotypic characterisation of cells within subcutaneous nodules. Arthritis Rheum 1983; 26: 1333-9.

6 Palmer D G, Hogg N, Allen C A, Highton J, Hessian P A. A mononuclear phagocyte subset associated with cell necrosis in rheumatoid nodules. Clin Immunol Immunopathol 1987; 45: 17-28.

7 Gardner D L. Rheumatoid arthritis. In: Pathological basis of the connective tissue diseases. London: Arnold, 1992: 485-9.

8 Edwards J C W. Structure of synovial lining. In: Henderson $\mathrm{B}$, Edwards $\mathrm{J} \mathrm{C} \mathrm{W}$, eds. The synovial lining in health and disease. London: Chapman and Hall, 1987: 17-39.

9 Wilkinson L S, Pitsillides A A, Worrall J G, Edwards J C W. Light microscopic characterisation of the fibroblastlike synovial intimal cell (synoviocyte). Arthritis Rheum 1992; 35: 1179-84

10 Wilkinson L S, Edwards J C W, Poston R, Haskard D O. Cell populations expressing VCAM-1 in normal and diseased synovium. Lab Invest 1993; 68: 82-8.

11 Morales Ducret J, Wayner E, Elices $M \mathrm{~J}$, et al. $\alpha_{4} / \beta_{1}$ integrin (VLA-4) ligands in arthritis: vascular cell adhesion molecule expression in synovium and on fibroblast-like molecule expression in synovium and on fib

12 Bröker B, Edwards J C W, Fanger M, Lydyard P. The prevalence and distribution of macrophages bearing FCRI, FCRII and FcRIII in synovium. Scand $\mathcal{F}$ Rheumatol 1990; 19: 123-35.

13 Franklin W A, Mason D Y, Pulford K, et al. Immunohistological analysis of human mononuclear phagocytes and dendritic cells by using monoclonal antibodies. Lab Invest 1986; 54: 322-6.

14 Mehdizadeh S, Bitensky L, Chayen J. The assay of uridine diphosphoglucose dehydrogenase activity: discrimination from xanthine oxidase activity. Cell Biochem Funct 1991; 9: $103-10$

15 Hoyhtya M, Myllyla R, Siuva J, Kivirkko K L, Tryggvason $\mathrm{K}$. Monoclonal antibodies to human prolyl-4K. Monoclonal antibodies to human
hydroxylase. Eur f Biochem 1984; 141:477-82

16 Wellicome S M, Thornhill M H, Pitzalis C, et al. A monoclonal antibody that detects a novel antigen on endothelial cells that is induced by tumour necrosis factor II-1 or lipopolysaccharide. F Immunol 1990; 144: 2558-65.

17 Sanchez-Madrid F, De Landazuri M O, Morago G, Cebrian M, Acevedo A, Bernabeu C. VLA-3; a novel polypeptide association within the VLA-molecular complex: cell distribution and biochemical characterisation. Eur $f$ Immunol 1986; 16: 1343-9.

18 Knapp P, ed. 4th leucocyte typing workshop. Oxford: Oxford University Press, 1989: 360, 1018 .

19 Pitsillides A A, Wilkinson L S, Mehdizadeh S, Bayliss M T, Edwards J C W. Uridine diphosphoglucose dehydrogenase activity in normal and rheumatoid synovium. $f$ Exp Pathol 1993; 74: 27-34

20 El-Gabalawy H, Wilkins J. $\beta 1$ (CD29) integrin expression in rheumatoid synovial membranes: an immunohistologic study of distribution patterns. $\mathcal{f}$ Rheumatol 1993; 20: $231-7$.

21 Wilkinson L S, Pitsillides A A, Edwards J C W. Comparison of synovial lining cells with palisading cells in rheumafoid nodule and surface cells in regenerated synovium [abstract] Br f Rheumatol 1993; 32 (suppl 1): 95.

22 Edwards J C W. The nature and origins of synovium: experimental approaches to the study of synoviocyte differentiation. $\mathcal{f}$ Anat. In press. 